\title{
Stark Resonances in dc fields from short time propagation of the field-free Hamiltonian
}

\author{
Nimrod Moiseyev ${ }^{(a)}$, Markus Glück and Jürgen H. Korsch ${ }^{(b)}$ \\ ${ }^{(a)}$ Department of Chemistry and Minerva Center for Non Linear Physics, Technion - Israel \\ Institute of Technology, Haifa 32000, Israel. \\ (b) Universität Kaiserslautern, Germany.
}

\begin{abstract}
A new method for calculating Stark resonances is presented and applied for illustration to the simple case of a one-particle, one-dimensional model Hamiltonian. The method is applicable for weak and strong dc fields. The only need, also for the case of many particles in multi-dimensional space, are either the short time evolution matrix elements or the eigenvalues and Fourier components of the eigenfunctions of the field-free Hamiltonian.
\end{abstract}

Typeset using REVTEX 
Recently there is an increasing experimental and theoretical effort in studying the lifetime of Stark resonances in highly excited Rydberg states [1-4]. In this case even a weak dc field must be considered as a strong perturbation. This is easy to see since the natural perturbation strength parameter is the product of the field strength and the localization length of the electron, divided by the very small first excitation energy of the electron in a highly excited Rydberg state. However, the increasing interest in Stark resonances is not limited to atomic and molecular physics. It arises also in other fields such as in microelectronics due to the important role of resonant tunneling in diodes, transistors and other coherent phenomena in semiconductors and mesoscopic systems [5].

Stark resonances can be obtained by different methods. All non-perturbative methods which have been developed so far solve the Schrödinger equation where the Hamiltonian consists of the field-free Hamiltonian together with the dc field term, $F x$. The method we present calculates the resonances from the time evolution of the field-free Hamiltonian $H_{0}$. As we will show below this method is closely related to a recent method developed by Glück, Kolovsky and Korsch [6].

Before we continue let us first introduce our approach for calculating Stark resonances. For the sake of simplicity, let us consider a one-dimensional system, represented by the field-free Hamiltonian, $H_{0}$, which is exposed to an external static field, $F x$ :

1. Calculate the field-free time-evolution operator

$$
\mathbf{U}_{0}(\tau)=\exp \left(-\mathrm{i} H_{0} \tau / \hbar\right)
$$

using plane waves $\langle x \mid n\rangle=L^{-1 / 2} \exp (2 \pi \mathrm{i} n x / L)$ as basis, up to time $\tau=2 \pi \hbar /(F L)$. The matrix elements of $\mathbf{U}_{0}(\tau)$ are denoted by $\left(U_{0}\right)_{n, n^{\prime}}$.

2. Construct a new matrix $\mathbf{U}$ by shifting the row index by one. That is,

$$
U_{n, n^{\prime}}=\left(U_{0}\right)_{n+1, n^{\prime}}
$$

Of course, if $\mathbf{U}_{\mathbf{0}}$ and $\mathbf{U}$ have a finite size, $-N \leq n, n^{\prime} \leq N$, the elements $U_{N, n^{\prime}}$ are 
equal to zero. One can avoid this row of zeros by taking the dimension of $\mathbf{U}$ smaller than the dimension of $\mathbf{U}_{0}$, i. e. $-N \leq n, n^{\prime} \leq N-1$.

3. Calculate the complex eigenvalues $\lambda=\exp \left(-i E_{\mathrm{res}} \tau / \hbar\right)$ of the non-unitary matrix $\mathbf{U}$. The real parts of the different values of $E_{\text {res }}$ are the resonance positions (modulo $F L$ ). The imaginary parts are associated with the widths.

Our strategy is as follows: First we will prove the above equations. Second, we will explain why complex values are obtained for $E_{\text {res }}$, i.e. from the eigenvalues of the matrix $\mathbf{U}$. Finally, we will give an illustrative numerical example.

\section{SPECTRA FROM FIELD-FREE PROPAGATION}

Here we prove that the spectra of the system in a dc field can be evaluated from the field-free time-evolution operator $H_{0}$ :

The time-evolution operator of the full Hamiltonian (with the dc field) can be approximately described by the split operator technique [7],

$$
\mathbf{U}(\tau)=e^{-\mathrm{i}\left(H_{0}+F x\right) \tau / \hbar} \approx e^{-\mathrm{i} F x \tau / \hbar} e^{-\mathrm{i} H_{0} \tau / \hbar}
$$

(as we will show below, in the present case and with the $\tau$ which we will define below, Eq.(3) is

equivalent to the symmetric split operator approach, i.e., $\left.\mathbf{U}(\tau) \approx e^{-\mathrm{i} F x \tau / 2 \hbar} e^{-\mathrm{i} H_{0} \tau / \hbar} e^{-\mathrm{i} F x \tau / 2 \hbar}\right)$. As $\tau$ gets smaller this approximation becomes more accurate. Therefore Eq. (3) is applicable only for short propagation time $\tau$.

Let us take plane waves

$$
\langle x \mid n\rangle=\frac{1}{\sqrt{L}} \exp (2 \pi \mathrm{i} n x / L)
$$

where $L$ is the box size, as a basis set for the $x$-coordinate. If the dimension of the system is bigger than one, for the other coordinates any orthogonal basis set can be used (for the sake of simplicity, we will skip the other coordinates and write down explicitly only the row and column indices associated with the $x$-coordinate). 
Given $F$ and $L$, the propagation time $\tau$ is taken as

$$
\tau=\frac{2 \pi \hbar}{F L}
$$

Thus, a large box size $L$ and therefore many Fourier basis functions are required for weak fields and a smaller box $L$ and a smaller number of basis functions are needed for stronger fields in order to get $\tau$ sufficiently small to yield converged results.

Within the Fourier basis, the time-evolution matrix is described as

$$
U_{n, n^{\prime}}=\sum_{k=-\infty}^{+\infty}\left\langle n\left|e^{-i F x \tau / \hbar}\right| k\right\rangle\left\langle k\left|e^{-i H_{0} \tau / \hbar}\right| n^{\prime}\right\rangle
$$

Recalling the definition of $\tau$, i.e. $F \tau / \hbar=2 \pi / L$, one immediately obtains

$$
U_{n, n^{\prime}}=\sum_{k=-\infty}^{+\infty} \delta_{n+1, k}\left(U_{0}\right)_{k, n^{\prime}}=\left(U_{0}\right)_{n+1, n^{\prime}}
$$

The eigenvalues of the matrix $\mathbf{U}$ are $\lambda=\exp \left(-\mathrm{i} E_{\text {res }} \tau / \hbar\right)$, where $E_{\text {res }}$ are the eigenvalues of the full Hamiltonian $H_{0}+F x$, as one can see from Eq. (3).

Following the same line of discussion, the symmetric split operator yields

$$
\left(U_{\text {sym }}\right)_{n, n^{\prime}}=\left(U_{0}\right)_{n+1 / 2, n^{\prime}-1 / 2}
$$

i.e. , to get the matrix elements of $\mathbf{U}_{\text {sym }}$, one first has to calculate $\mathbf{U}_{\mathbf{0}}$ within the Bloch index shifted basis set $\langle x \mid n-1 / 2\rangle=L^{-1 / 2} \exp (2 \pi \mathrm{i}(n-1 / 2) x / L)$, and later on, as before, shift the row index by one. Thus, the symmetric split operator within the basis $\langle x \mid n\rangle$ yields the same matrix as the non-symmetric split operator within the basis $\langle x \mid n-1 / 2\rangle$, and, therefore, both approaches are equivalent.

When the eigenfunctions $\psi_{j}^{(0)}$ and the corresponding eigenvalues $E_{j}^{(0)}$ of the field-free Hamiltonian $H_{0}$ are known, we can use $\psi_{j}^{(0)}$ to represent the time evolution operator. The time-evolution matrix in this new basis set is given by

$$
\mathcal{U}=\mathbf{C}^{\dagger} \mathbf{U C}
$$

with $C_{n, j}=\left\langle n \mid \psi_{j}^{(0)}\right\rangle$. Therefore, the matrix elements of $\mathcal{U}$ are given by 


$$
\mathcal{U}_{j, j^{\prime}}=e^{-i E_{j^{\prime}}^{(0)} \tau / \hbar} \sum_{n} C_{n, j}^{*} C_{n+1, j^{\prime}} .
$$

Again the eigenvalues of the full Hamiltonian which includes the dc field are $E_{\text {res }}=\mathrm{i} \hbar / \tau \ln \lambda$.

\section{COMPLEX EIGENVALUES BY TRUNCATING U}

In this section, we show how complex eigenvalues of the full Hamiltonian, $E=E_{r}-\mathrm{i} \Gamma / 2$, can be obtained by truncating the infinite time evolution matrix $\mathbf{U}$.

While the infinite matrix $\mathbf{U}$ is unitary, it becomes non-unitary, if we represent it within a finite basis $-N \leq n, n^{\prime} \leq N$. Since the finite basis representation of the field-free time evolution matrix $\mathbf{U}_{\mathbf{0}}$ is unitary, from Eqs. (6) and (7) one can see that the non-unitarity arises from the multiplication (from the left) of $\mathbf{U}_{0}$ by a finite matrix $\mathbf{B}$,

$$
B_{n, n^{\prime}}=\left\langle n|\exp (-2 \pi \mathrm{i} x / L)| n^{\prime}\right\rangle
$$

with matrix elements $B_{n+1, n}=1$ and all others equal to zero. The truncated matrix $\mathbf{B}$ is ill defined since

$$
\exp (-\mathrm{i} F x \tau / \hbar)|n\rangle=\exp (-2 \pi \mathrm{i} x / L)|n\rangle=|n-1\rangle
$$

is not in the domain of $\mathbf{B}$ when $n=-N$. Therefore, $\mathbf{B}$ is non-unitary, i.e.

$$
\mathbf{B}^{\dagger} \mathbf{B}=\mathbf{I}-\mathbf{P}_{-\mathbf{N}},
$$

where $\mathbf{I}$ is the unit matrix and $\mathbf{P}_{-\mathbf{N}}$ is the matrix of the projection operator $P_{-N}=|-N\rangle\langle-N|$ on the state $|-N\rangle$. Consequently, the finite matrix representation of $\mathbf{U}$, which is the product of a unitary finite matrix $\mathbf{U}_{\mathbf{0}}$ and a non-unitary finite matrix $\mathbf{B}$, is a non-unitary matrix.

The matrix $\mathbf{B}$ does not have a complete spectrum, i.e. the number of linearly independent eigenvectors is smaller than the dimension of the matrix $(2 N+1$ in our case) [8]. In our

special case, $\mathbf{B}$ has only one (linearly independent) eigenvector $(1,0,0, \ldots, 0)$ for any $N$. The corresponding eigenvalue is zero. On the basis of the discussion in Ref. [9] it 
is clear that by adding a small number $\epsilon$ to all matrix elements of $\mathbf{B}, 2 N+1$ linearly independent eigenvectors will be obtained, associated with non-degenerate eigenvalues which are proportional to $\epsilon^{1 /(2 N+1)}$. As $\epsilon$ is reduced to zero all eigenvalues will approach the value zero, accompanied by the coalescence of the $2 N+1$ eigenvectors of the distorted matrix $\mathbf{B}$.

One can carry out a transformation of $\mathbf{B}$, for example by using the eigenfunctions of the field-free Hamiltonian as a basis set. The new transformed matrix $\mathcal{B}$ is given by

$$
\mathcal{B}=\mathbf{C}^{\dagger} \mathbf{B C} \text {. }
$$

Although $\mathcal{B}$ does have a complete spectrum, unlike $\mathbf{B}$, it is still a non-unitary matrix, since

$$
\mathcal{B}^{\dagger} \mathcal{B}=\mathbf{C}^{\dagger}\left(\mathbf{I}-\mathbf{P}_{-\mathbf{N}}\right) \mathbf{C}=\mathbf{I}-\mathbf{C}^{\dagger} \mathbf{P}_{-\mathbf{N}} \mathbf{C}
$$

Therefore the matrix $\mathcal{U}=\mathcal{B} \mathcal{U}_{0}^{(d)}$, where $\mathcal{U}_{0}^{(d)}$ is the diagonal field-free time evolution matrix, is non-unitary either. Consequently, the eigenvalues of $\mathcal{U}$, which are denoted as $\lambda=\exp \left(-\mathrm{i} E_{\text {res }} \tau / \hbar\right)$, are associated with complex values of $E_{\text {res }}$, whereas the eigenvalues of the infinite time-evolution matrix $\mathbf{U}_{\infty}(\tau)$ (see Eq. (6)) are real!

Since B is given by the matrix elements of the operator $B=\exp (-2 \pi \mathrm{i} x / L)$, it is natural to ask for the connection to the Hermitian operator $\mathbf{X}$, the matrix of which is given by $X_{n, n^{\prime}}=\left\langle n|x| n^{\prime}\right\rangle=\frac{L(-1)^{n-n^{\prime}}}{2 \pi \mathrm{i}\left(n-n^{\prime}\right)}$ for $n^{\prime} \neq n$ and $X_{n, n}=0$. It is easy to see that

$$
\mathbf{B} \neq \exp (-2 \pi \mathrm{i} \mathbf{X} / L)
$$

since the matrix on the right side is clearly unitary and $\mathbf{B}$ is non-unitary. Furthermore, it is not possible to construct a matrix, the exponential of which is $\mathbf{B}$, since the logarithm of $\mathbf{B}$ is not defined. Nevertheless, one can take the matrix $\mathbf{D}$ which consists of the eigenvectors of $\mathbf{X}$, express $\mathbf{B}$ using this basis and calculate the eigenvalues of $\mathcal{B}=\mathbf{D}^{\dagger} \mathbf{B D}$. In this case one gets a set of eigenvalues $\lambda_{j}=\exp \left(-2 \pi \mathrm{i} z_{j} / L\right)$, where the $z_{j}$ are approximately embedded on a horizontal line in the lower complex plane.

Therefore, we may take the following conclusion: The time evolution matrix $\mathbf{U}$ is the exponent of the full finite Hamiltonian matrix (times $-\mathrm{i} \tau / \hbar$ ). This exponent is a unitary 
operator, but the truncation described above leads to a non-hermitian matrix representation of the full Hamiltonian [10]. The eigenvalues of the non-unitary matrix $\mathbf{U}$ or $\mathcal{U}$ denoted by $\exp (-\mathrm{i} E \tau / \hbar)$ provide complex values for $E$ which are the eigenvalues of the non-hermitian full Hamiltonian. Our numerical studies (see next section) show very clearly and with no doubt that the real and imaginary parts of these complex values of $E$ are associated with the energy and width of the Stark resonances, respectively.

\section{ILLUSTRATIVE EXAMPLE}

As an example, let us take the one-dimensional model Hamiltonian

$$
H_{0}=\frac{p^{2}}{2}-4.5 \exp \left(-x^{2}\right)
$$

which is exposed to the external field $F x$ with a field strength $F=1(\hbar=1)$. This model has been used by Reinhardt and his coworkers as a test case for the use of complex scaling and complex translation for calculating Stark resonances [11].

From $F=1$ and $\hbar=1$ we get $\tau=2 \pi / L$ (note that the definition of $\tau=2 \pi \hbar /(F L)$ is the only part where the field strength enters. Therefore, by modifying $\tau$, the algorithm can be easily adjusted to yield the resonances for a different field strength $F$ ). The calculation scheme is as follows: For every box size $L$ we take a sufficiently large number of Fourier basis states, solve the time-independent eigenvalue problem $H_{0} \psi_{j}=E_{j} \psi_{j}$ and calculate $\mathbf{U}$ according to equation (10). The eigenvalues $\lambda$ of $\mathbf{U}$ yield the resonance energies via $E=\mathrm{i} \hbar / \tau \log (\lambda)$.

Table I shows results for the Stark resonance associated with the ground state, i.e. the eigenvalue with the smallest imaginary part. The resonance position and width converge to the exact values when the box size $L$ is increased. From the definition of $\tau$ given above one can see that increasing the box size $L$ implies reducing the propagation time $\tau$ of the field-free Hamiltonian.

Let us conclude: We presented a new method to calculate Stark resonances from short 
time propagation of the field-free Hamiltonian. The numerical realization of the method is extremely simple. The method can be easily extended to higher dimensions.

The question is why the matrix truncation as described above provides not only complex eigenvalues, but the Stark resonances. A possible conjecture is that the truncation of the matrix B, as described above, has the same effect as the translation of the coordinate $x$ into the complex plane, which is known [12], to make the complex divergent Stark resonance wavefunctions to be square integrable and decay to zero as the coordinate $x$ is taken to infinity (in the limit $L \rightarrow \infty$ ).

This conjecture is based on the fact, that the operator $\exp (-\mathrm{i} F x \tau / \hbar)$ is a shift operator in momentum space, i.e. $\exp (-\mathrm{i} F x \tau / \hbar)|n\rangle=\exp (-2 \pi \mathrm{i} x / L)|n\rangle=|n-1\rangle$. It is clear that by a truncation of the matrix to a finite dimension, $-N \leq n \leq N$, we do not let the shift operator transfer the particle to a momentum which is smaller than $-2 \pi N \hbar / L$. The only way one can get converged results (within these "constraints") is when the quantum particle is localized in the momentum space and it is not "kicked" by the force of the dc field to get momenta exceeding this value. This happens if and only if the quantum particle is trapped in a metastable resonance state (note that the Husimi distribution functions of the complex scaled resonance states show localization in the classical phase space). This rational is in principle very similar to the one which stands behind our previous explanation [13] (see also the related method proposed very recently for calculating Wannier-Bloch resonance states $[6])$.

The application of this method to the calculation of the 3D Stark resonances of hydrogen atoms excited to high Rydberg states is currently investigated.

\section{ACKNOWLEDGMENTS}

This work was supported in part by the Israel-US Binational Science Foundation and by the Fund of promotion of research at the Technion. Major part of this work has been carried out during the stay of NM in Kaiserslautern as an Alexander von Humboldt awardee. 


\section{REFERENCES}

[1] W. A. Chupka, J. Chem. Phys. 98, 4520 (1993); ibid, 99, 5800 (1993).

[2] M. Bixon and J. Jortner, J. Chem. Phys. 105, 1363 (1996); ibid, 103, 4431 (1995).

[3] A. Muhlpfordt, U. Even, E. Rabani, and R. D. Levine, Phys. Rev. A 51, 3922 (1995).

[4] P. Bellome, D. Farrelly, and T. Uzer, J. Chem. Phys. 108, 5295 (1998).

[5] M. Wagner, Phys. Rev. B 57, 11899 (1998); L. Yumpeng and L. Enze, Appl. Surf. Sci. 87 (1995); D. Farrelly, E. Lee, and T. Uzer, Phys. Rev. Lett. 80, 3884 (1998) and refereces therein; P. Bellome, D. Farrelly and T. Uzer, J. Chem. Phys. 108, 5295 (1998).

[6] M. Glück, A. Kolovsky, and H. J. Korsch, J. Phys. A (submitted for publication); Phys.Lett. A (in press).

[7] M. D. Feit, J. D. Fleck and A. Steiger, J. Comp. Phys. 47, 416 (1982); C. Leforestier. R. H. Bisseling, C. Cerjan, M. D. Feit, R. Friesner, A. Guldberg, A. Hammerich, G. Jolicard, W. Karrlein, H.-D. Meyer, N. Lipkin, O. Roncero, and R. Kosloff, J. Comp. Phys. 94, 59 (1994).

[8] J. H. Wilkinson: The algebraic eigenvalue problem (Claredon Press, Oxford, 1965).

[9] N. Moiseyev, Phys. Rep. C 302, 211 (1998).

[10] For related studies of problems arising from the truncation of infinite matrices which represent operators, see O. E. Alon, N. Moiseyev, and A. Peres, J. Phys. A 28, 1765 (1995).

[11] C. Cerjan, R. Hedges, W. P. Reinhardt, K. Scheibner, and J. J. Wendoloski, Intern. J. Quantum Chem. 14, 393 (1978).

[12] C. A. Nicolaides and S. I. Themelis, Phys. Rev. A 45, 349 (1992), see also J. E. Avron, and I. W. Herbst, Commun. Math. Phys. 52, 239 (1977). 
[13] M. Glück, A. Kolovsky, H. J. Korsch, and N. Moiseyev, EPJ D (in press). 


\section{TABLES}

TABLE I. Stark resonances $E_{r}-\mathrm{i} \Gamma / 2$ associated with the ground state of the $1 \mathrm{D}$ model Hamiltonian $H_{0}$ (Eq. 17) in the external field $F x$ with $F=1$ and $\hbar=1$. The box size $L$ is varied and for any value of $L$ the resonance is calculated as described in the text. Also given is the complex scaling result which is considered as exact. Note that increasing $L$ is equivalent to the reduction of the propagation time $\tau$ (see Eq. (5)) of the field-free Hamiltonian.

\begin{tabular}{rcc}
\hline \hline$L / 2 \pi$ & $-E($ a.u. $)$ & $10^{4} \Gamma($ a.u. $)$ \\
\hline 20 & 3.29744 & 9.495 \\
40 & 3.29773 & 8.992 \\
60 & 3.29778 & 8.931 \\
80 & 3.29780 & 8.926 \\
100 & 3.29781 & 8.939 \\
\hline exact & 3.29783 & 8.934 \\
\hline \hline
\end{tabular}

